\title{
Erratum: Generation of chiral asymmetry via helical magnetic fields [Phys. Rev. D 101, 103028 (2020)]
}

\author{
Jennifer Schober®, Tomohiro Fujita, and Ruth Durrer
}

Q (Received 14 February 2022; published 2 March 2022)

DOI: 10.1103/PhysRevD.105.069901

This is an erratum to the paper "Generation of chiral asymmetry via helical magnetic fields", published in Phys. Rev. D 101, 103028 (2020).

In Figs. 10(d) and 10(h) of this publication, the labels indicate that the kinetic energy spectra $E_{\text {kin }}(k)$ are presented for runs R8mhd and R8, respectively. However, the data shown in Figs. 10(d) and 10(h) corresponds to the magnetic energy spectra $E_{\text {mag }}(k)$.

We present $E_{\text {mag }}(k)$ and $E_{\text {kin }}(k)$ for runs R8mhd and R8 in Fig. 1 . In comparison to $E_{\text {mag }}(k)$, the kinetic energy spectra generally span over a wider range of wave numbers $k$ and have a lower amplitude.

The error in the original publication does not affect its conclusion.
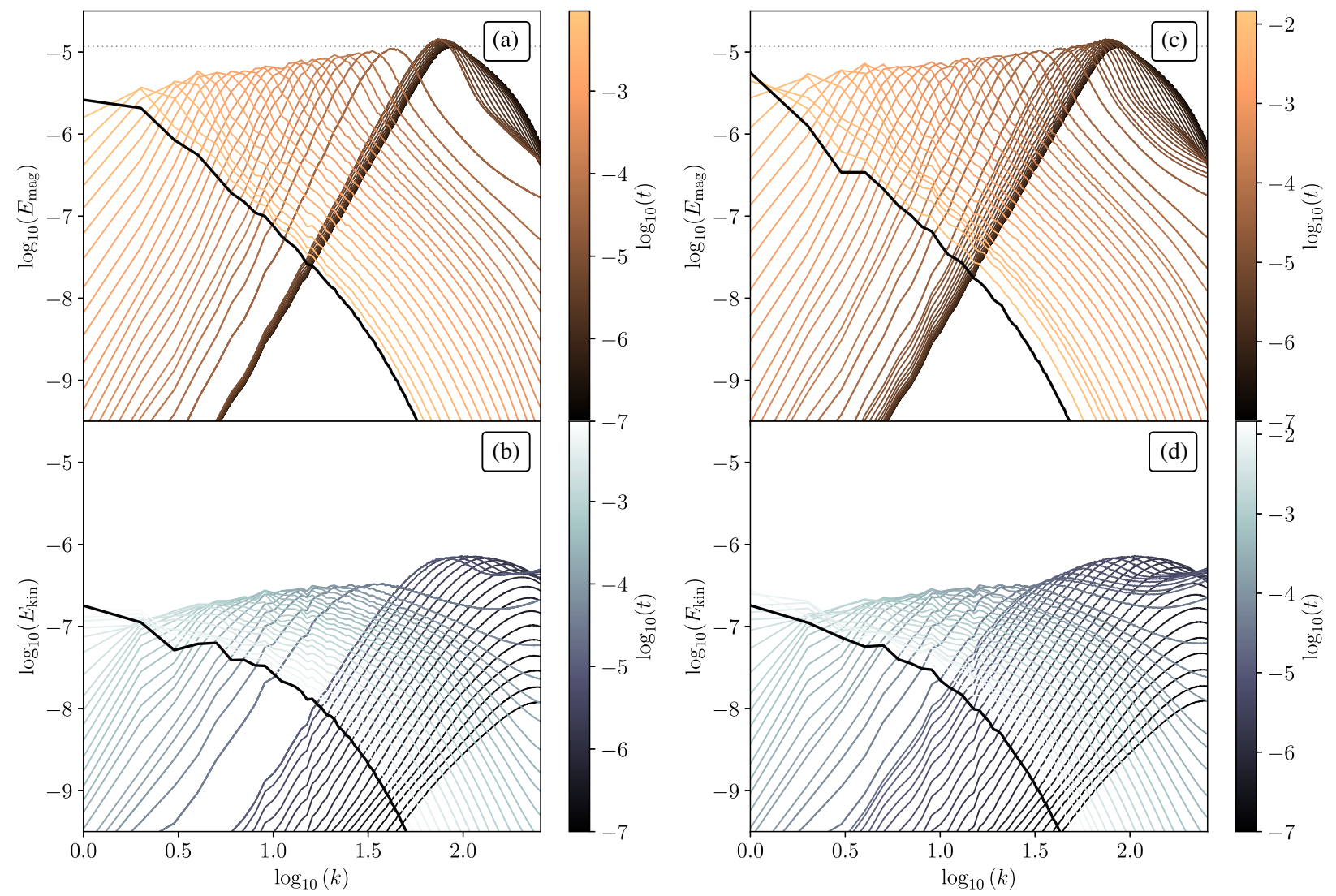

FIG. 1. Comparison of the magnetic (upper panels) and kinetic energy spectra (lower panels) in a run with classical MHD (left panels, run R8mhd) and a run with chiral MHD (right panels, run R8). 